\title{
CLOSURE OF DUODENAL PERFORATION BY GASTRIC SEROMUSCULAR ADVANCEMENT FLAP
}

\author{
Periasamy Subbiah', Gayathri², Gopinath ${ }^{3}$, Sivan', Praveen Kumar ${ }^{5}$, Prashanth ${ }^{6}$, Reka G $^{7}$
}

1 Professor, Department of Surgery, CMCH, Chengalpattu, Tamil Nadu.

${ }^{2}$ Associate Professor, Department of Surgery, CMCH, Chengalpattu, Tamil Nadu.

${ }^{3}$ Assistant Professor, Department of Surgery, CMCH, Chengalpattu, Tamil Nadu.

${ }^{4}$ Assistant Professor, Department of Surgery, CMCH, Chengalpattu, Tamil Nadu.

5Postgraduate, Department of Surgery, CMCH, Chengalpattu, Tamil Nadu.

${ }^{6}$ Postgraduate, Department of Surgery, CMCH, Chengalpattu, Tamil Nadu.

${ }^{7}$ Postgraduate, Department of Surgery, CMCH, Chengalpattu, Tamil Nadu.

\section{ABSTRACT}

\section{BACKGROUND}

Perforation remains a surgical disease and management means emergency surgical intervention. Primary closure with omental buttress, Graham's live/dead omental patch repair, Jejunal serosal (Thal) patch repair and antrectomy with Billroth II reconstruction have been the traditionally followed procedures depending on the site/size of the perforation. This study reports a series of cases of perforated duodenal ulcer closed by Gastric seromuscular advancement flap. This article compares the advantages and disadvantages of either methods and details the indications and method of this alternative repair.

\section{METHOD}

Emergency exploratory laparotomy of cases of duodenal ulcer perforation.

\section{RESULTS}

Study group of 112 cases with similar survival outcome in uncomplicated cases with added advantage of improved survival outcome in complicated cases with unhealthy or absent omentum.

\section{CONCLUSION}

This article takes into account a total of 112 cases of perforated ulcer operated at our institution from 2011-2015 with 50 cases being operated by the method under study. Results with seromuscular flap closure were comparable with that of other methods and more beneficial in the setting of unavailable or contaminated/unhealthy omentum.

\section{KEYWORDS}

Duodenal Ulcer, Perforation, Batch, Omentum, Flap, Seromuscular Layer.

HOW TO CITE THIS ARTICLE: Subbiah P, Gayathri, Gopinath, et al. Closure of duodenal perforation by gastric seromuscular advancement flap. J. Evolution Med. Dent. Sci. 2016;5(73):5355-5358, DOI: 10.14260/jemds/2016/1215

\section{INTRODUCTION}

The perforation is usually in the first portion of the duodenum and can easily be accessed through an upper midline incision. Perforations smaller than $1 \mathrm{~cm}$ can generally be closed primarily and buttressed with well-vascularised omentum. For larger perforations, a Graham patch repair with a tongue of healthy omentum is performed. For very large perforations $(>3 \mathrm{~cm})$, control of the duodenal defect can be difficult. The defect should be closed by the application of healthy tissue, such as omentum or jejunal serosa, with placement of duodenostomy tube and wide bore drainage tube. This is likely to result in leakage of GI contents into the drain, but in most cases sepsis will resolve. An alternative in this difficult situation is antrectomy and a Billroth II reconstruction.

\section{AIM \& OBJECTIVES}

To study the efficacy of Gastric seromuscular flap closure of

Financial or Other, Competing Interest: None.

Submission 31-07-2016, Peer Review 28-08-2016,

Acceptance 02-09-2016, Published 10-09-2016.

Corresponding Author:

Dr. Periasamy Subbiah,

U. S. Hospital 7, Balakumaran Nagar,

Part-C, Kolathur,

Chennai-99.

E-mail: drperiasamys@yahoo.com

DOI: $10.14260 /$ jemds/2016/1215

\section{(c) (i) $९$}

Duodenal ulcer perforation over omental patch (Live/dead) in setting of unhealthy/absent omentum. Survival analysis of patients treated by either methods.

The outcome of patients with a perforated ulcer depends on the following:

1. Delay from initial evaluation to treatment: recent data shows a delay in surgical treatment, in part because of more extensive diagnostic work-up.

2. Site of perforation: gastric perforations are associated with a poor prognosis.

3. Patient's age: elderly patients, who often have associated comorbid conditions, have a poor outcome.

4. Presence of hypotension at initial evaluation (Systolic blood pressure $<100 \mathrm{~mm}$ of $\mathrm{Hg}$ ) leads to poor results.

The seromuscular advancement flap can be of much help in the following situations: cases with extensive peritoneal contamination leading to unhealthy omentum making it unsuitable for aiding closure; cases where there is nonavailability of omentum due to prior surgical interventions.

\section{MATERIALS AND METHOD OF STUDY}

Emergency exploratory laparotomy of suspected cases of duodenal ulcer perforation.

\section{Operative Method}

Abdomen was opened by an upper midline incision (preferably upper midline). After identifying the 


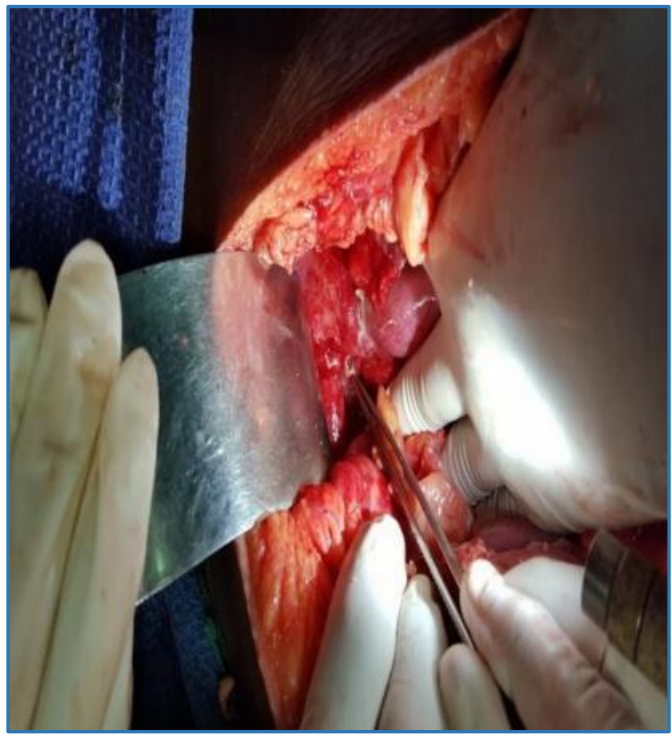

Fig. 1: Site of Perforation

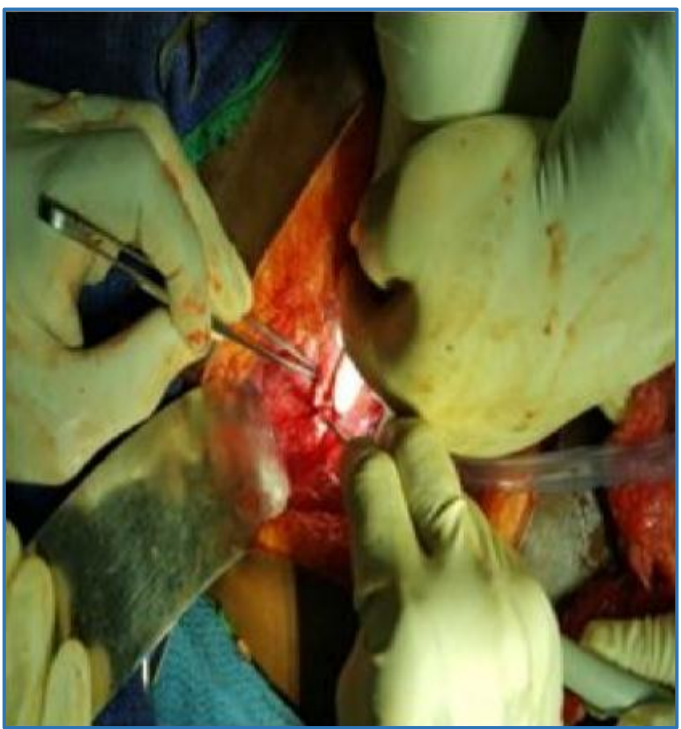

Fig. 2: Seromuscular Flap Raised

Site of perforation, Seromuscular flap is raised adjacent to the perforated site taking care to raise appropriate thickness without breaching the submucosa.

The flap is then rotated/advanced to superimpose upon the perforation and sutured with 2-0 Vicryl.

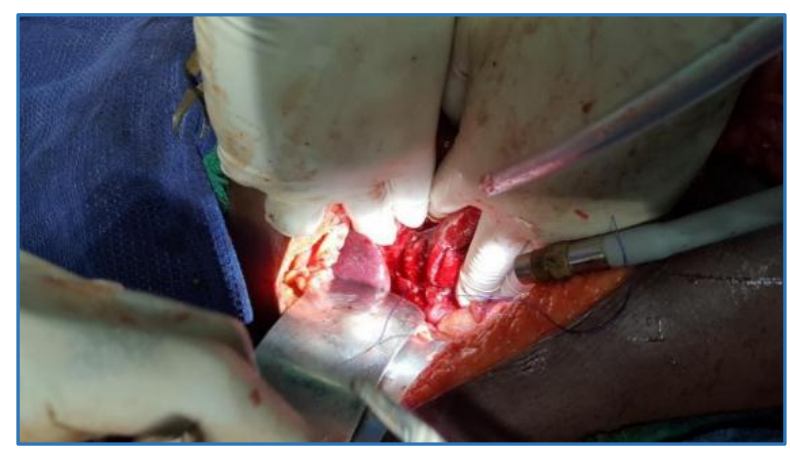

Fig. 3: Flap Sutured over Perforation

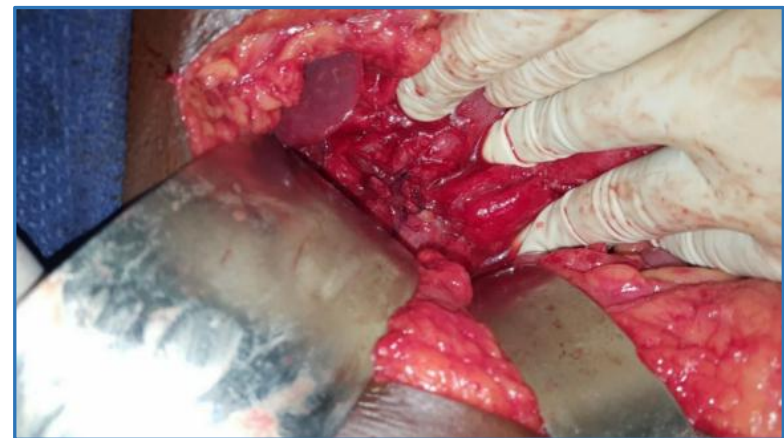

Fig. 4: After the Closure of Perforation with Flap

Disadvantages - Surgical expertise cannot be used for large perforations.

\section{Method of Study}

A total of 112 cases of perforated duodenal ulcer operated at our institution from 2011-2016 were included in the study with 50 cases being operated by the method under study.

A prospective randomised trial of gastric seromuscular advancement flap closure versus omental patch closure of perforated duodenal ulcer was done.

Two comparative studies done using the same study group. One under the setting of healthy omentum. Other study done under setting of unhealthy or absent omentum. Both studies compared the efficacy/mortality of seromuscular flap versus omental patching.

\section{RESULTS (DATA ANALYSIS)}

\section{Following Factors were taken into Consideration}

- Comorbid conditions/age,

- Presence/absence of sepsis,

- Systolic BP at presentation,

- Size of perforation.

- Omental condition (Unhealthy/necrosis/absence).

Total of 112 cases of Duodenal ulcer perforation were selected (Leaving aside gastric \& large DU perforations) of which cases with healthy omentum were 70 and unhealthy/absent omentum were 42 .

Out of the 70 cases with healthy omentum, 45 cases were operated by Omental patch closure and 22 were operated by GSM flap.

Out of the 42 cases with unhealthy/absent omentum, 14 cases were operated by Omental patch closure and 28 were operated by GSM Flap.

Survival analysis done amongst two groups comparing the two types of perforation closure method. Statistical analysis done. Chi-square tests done.

\section{Frequency Table}

\begin{tabular}{|c|c|c|}
\hline & Frequency & Percent \\
\hline Valid Male & 99 & 88.4 \\
\hline Female & 13 & 11.6 \\
\hline Total & $\mathbf{1 1 2}$ & $\mathbf{1 0 0 . 0}$ \\
\hline \multicolumn{3}{|c|}{ Sex Distribution } \\
\hline
\end{tabular}




\begin{tabular}{|c|c|c|c|c|c|}
\hline \multirow{3}{*}{ Sex } & & \multicolumn{2}{|c|}{ Age Category } & & \multirow{2}{*}{ Total } \\
\cline { 2 - 5 } & $\begin{array}{c}\mathbf{1 5 - 3 0} \\
\text { Yrs. }\end{array}$ & $\begin{array}{c}\mathbf{3 1 - 4 5} \\
\text { Yrs. }\end{array}$ & $\begin{array}{c}\mathbf{4 6 - 6 0} \\
\text { Yrs. }\end{array}$ & $\begin{array}{c}\text { Y60 } \\
\text { Yrs. }\end{array}$ & \\
\hline Male & 25 & 29 & 36 & 9 & 99 \\
Female & 3 & 3 & 4 & 3 & 13 \\
Total & 28 & 32 & 40 & 12 & 112 \\
\hline \multicolumn{6}{|c|}{ Sex Age Category Cross Tabulation } \\
\hline
\end{tabular}

\begin{tabular}{|c|c|c|}
\hline & Age & Follow-up \\
\hline N Valid & 112 & 112 \\
\hline Missing & 0 & 0 \\
\hline Mean & 44.36 & 56.95 \\
\hline Median & 45.00 & 60.00 \\
\hline Std. Deviation & 14.371 & 27.859 \\
\hline \multicolumn{3}{|c|}{ Statistics } \\
\hline
\end{tabular}

\begin{tabular}{|c|c|c|}
\hline Valid & Frequency & Percent \\
\hline Present & 19 & 17.0 \\
\hline Absent & 93 & 83.0 \\
\hline Total & $\mathbf{1 1 2}$ & $\mathbf{1 0 0 . 0}$ \\
\hline \multicolumn{3}{|c|}{ Comorbidity } \\
\hline
\end{tabular}

\begin{tabular}{|c|c|c|}
\hline Valid Omentum & Frequency & Percent \\
\hline Healthy & 70 & 62.5 \\
\hline Unhealthy & 42 & 37.5 \\
\hline Total & $\mathbf{1 1 2}$ & $\mathbf{1 0 0 . 0}$ \\
\hline \multicolumn{2}{|c|}{ Condition of Omentum } \\
\hline
\end{tabular}

\begin{tabular}{|c|c|c|}
\hline & Frequency & Percent \\
\hline Valid Omentum & 62 & 55.4 \\
\hline GSM Flap & 50 & 44.6 \\
\hline Total & $\mathbf{1 1 2}$ & $\mathbf{1 0 0 . 0}$ \\
\hline \multicolumn{2}{|c|}{ Material for Surgery } \\
\hline
\end{tabular}

\begin{tabular}{|c|c|c|}
\hline & Frequency & Percent \\
\hline Valid Alive & 97 & 86.6 \\
\hline Dead & 15 & 13.4 \\
\hline Total & $\mathbf{1 1 2}$ & $\mathbf{1 0 0 . 0}$ \\
\hline \multicolumn{3}{|c|}{ Status after Surgery } \\
\hline
\end{tabular}

\begin{tabular}{|c|c|c|}
\hline & Frequency & Percent \\
\hline Valid 15-30 Yrs. & 28 & 25.0 \\
\hline 31-45 Yrs. & 32 & 28.6 \\
\hline $46-60$ Yrs. & 40 & 35.7 \\
\hline$>60$ Yrs. & 12 & 10.7 \\
\hline Total Age Distribution & $\mathbf{1 0 0 . 0}$ \\
\hline \multicolumn{2}{|c}{} \\
\hline
\end{tabular}

\begin{tabular}{|c|c|c|c|c|c|}
\hline \multirow{3}{*}{ Comorbidity } & \multicolumn{3}{|c|}{ Age Category } & & \multirow{2}{*}{ Total } \\
\cline { 2 - 6 } & $\begin{array}{c}\mathbf{1 5 - 3 0} \\
\text { Yrs. }\end{array}$ & $\begin{array}{c}\mathbf{3 1 - 4 5} \\
\text { Yrs. }\end{array}$ & $\begin{array}{c}\mathbf{4 6 - 6 0} \\
\text { Yrs. }\end{array}$ & $\begin{array}{c}>60 \\
\text { Yrs. }\end{array}$ & \\
\hline Present & 0 & 2 & 7 & 10 & 19 \\
\hline Absent & 28 & 30 & 33 & 2 & 93 \\
\hline Total & $\mathbf{2 8}$ & $\mathbf{3 2}$ & $\mathbf{4 0}$ & $\mathbf{1 2}$ & $\mathbf{1 1 2}$ \\
\hline \multicolumn{7}{|c|}{ Comorbidities* Age Category Cross Tabulation } \\
\hline
\end{tabular}

\begin{tabular}{|c|c|c|c|c|}
\hline \multicolumn{5}{|c|}{ Cross Tab } \\
\hline \multicolumn{2}{|c|}{ Count } & \multicolumn{2}{c|}{ Survival } & \multirow{2}{*}{ Total } \\
\cline { 3 - 4 } & Alive & Dead & \\
\hline Omental & Healthy & 64 & 6 & 70 \\
\hline Condition & Unhealthy & 33 & 9 & 42 \\
\hline \multicolumn{4}{|c|}{ Omental Condition* Survival } \\
\hline
\end{tabular}

\begin{tabular}{|c|c|c|c|c|c|c|}
\hline & Value & Df & $\begin{array}{c}\text { Asymp. } \\
\text { Sig. } \\
\text { (2-Sided) }\end{array}$ & $\begin{array}{l}\text { Exact Sig. } \\
\text { (2-Sided) }\end{array}$ & $\begin{array}{c}\text { Exact Sig. } \\
\text { (1-Sided) }\end{array}$ \\
\hline $\begin{array}{c}\text { Pearson } \\
\text { Chi-square }\end{array}$ & $3.741 \mathrm{a}$ & 1 & .052 & & \\
\hline $\begin{array}{c}\text { Continuity } \\
\text { Correction }\end{array}$ & 2.715 & 1 & .099 & & \\
\hline $\begin{array}{c}\text { Likelihood } \\
\text { Ratio }\end{array}$ & 3.612 & 1 & .057 & & .052 \\
\hline $\begin{array}{c}\text { Fisher's } \\
\text { Exact Test }\end{array}$ & 112 & & & & & \\
\hline $\begin{array}{c}\text { N of Valid } \\
\text { Cases }\end{array}$ & & & & & \\
\hline \multicolumn{7}{c|}{ Chi-square Tests } \\
\hline
\end{tabular}

a. 0 cells $(.0 \%)$ have expected count less than 5 . The minimum expected count is 5.63 .

b. Computed only for a $2 \times 2$ table.

\begin{tabular}{|c|c|c|c|c|}
\hline \multicolumn{5}{|c|}{ Crosstab } \\
\hline \multirow{2}{*}{\multicolumn{2}{|c|}{ Count }} & \multicolumn{2}{|c|}{ Survival } & \multirow[b]{2}{*}{ Total } \\
\hline & & Alive & Dead & \\
\hline \multirow{2}{*}{ Surgery } & Omentum & 50 & 12 & 62 \\
\hline & Flap & 47 & 3 & 50 \\
\hline \multicolumn{2}{|c|}{ Total } & 97 & 15 & 112 \\
\hline \multicolumn{5}{|c|}{ Surgery* Survival } \\
\hline
\end{tabular}

\begin{tabular}{|c|c|c|c|c|c|c|}
\hline & Value & Df & & $\begin{array}{c}\text { Asymp. } \\
\text { Sig. } \\
\text { (2-sided) }\end{array}$ & $\begin{array}{c}\text { Exact } \\
\text { Sig. } \\
\text { (2-sided) }\end{array}$ & \begin{tabular}{|c|} 
Exact \\
Sig. \\
(1-sided)
\end{tabular} \\
\hline $\begin{array}{c}\text { Pearson } \\
\text { Chi-Square }\end{array}$ & $4.256^{\mathrm{a}}$ & & 1 & .039 & & \\
\hline $\begin{array}{l}\text { Continuity } \\
\text { Correction }^{b}\end{array}$ & 3.182 & & 1 & .074 & & \\
\hline $\begin{array}{l}\text { Likelihood } \\
\text { Ratio }\end{array}$ & 4.587 & & 1 & .032 & & \\
\hline $\begin{array}{c}\text { Fisher's } \\
\text { Exact Test }\end{array}$ & & & & & .051 & .034 \\
\hline $\begin{array}{c}\mathrm{N} \text { of Valid } \\
\text { Casesb }^{\mathrm{b}}\end{array}$ & 112 & & & & & \\
\hline \multicolumn{7}{|c|}{ quare Tests } \\
\hline
\end{tabular}

a. 0 cells $(.0 \%)$ have expected count less than 5 . The minimum expected count is 6.70 .

b. Computed only for a $2 \times 2$ table.

\begin{tabular}{|c|c|c|c|}
\hline \multirow{4}{|c|}{ Count } \\
\hline \multirow{2}{*}{ Age Category } & \multicolumn{2}{|c|}{ Survival } & \multirow{2}{*}{ Total } \\
\cline { 2 - 3 } & Alive & Dead & \\
\hline $15-30$ Yrs. & 28 & 0 & 28 \\
\hline $31-45$ Yrs. & 30 & 2 & 32 \\
\hline $46-60$ Yrs. & 35 & 5 & 40 \\
\hline$>60$ Yrs. & 4 & 8 & 12 \\
\hline Total & $\mathbf{9 7}$ & $\mathbf{1 5}$ & $\mathbf{1 1 2}$ \\
\hline \multicolumn{2}{|c|}{ Age Category Survival Cross Tabulation } \\
\hline
\end{tabular}




\begin{tabular}{|c|c|c|c|c|}
\hline & Value & Df & & $\begin{array}{c}\text { Asymp. Sig. } \\
\text { (2-sided) }\end{array}$ \\
\hline $\begin{array}{c}\text { Pearson Chi- } \\
\text { square }\end{array}$ & $\begin{array}{c}35.127 \\
\text { a }\end{array}$ & & 3 & .000 \\
\hline Likelihood Ratio & 27.828 & & 3 & .000 \\
\hline N of Valid Cases & 112 & & & Chi-square Tests \\
\hline \multicolumn{6}{|c|}{}
\end{tabular}

3 cells $(37.5 \%)$ have expected count less than 5 . The minimum expected count is 1.61 .

\begin{tabular}{|c|c|c|c|}
\hline & Chi-square & df & Sig. \\
\hline $\begin{array}{c}\text { Log Rank } \\
\text { (Mantel-Cox) }\end{array}$ & 4.122 & 1 & .042 \\
\hline \multicolumn{3}{|c|}{ Overall Comparisons } \\
\hline
\end{tabular}

Test of equality of survival distributions for the different levels of surgery.

\begin{tabular}{|c|c|c|c|c|}
\hline \multirow{3}{*}{ Surgery } & \multirow{3}{*}{ Estimate } & \multirow{3}{*}{$\begin{array}{c}\text { Std. } \\
\text { Error }\end{array}$} & \multirow{2}{*}{\multicolumn{2}{|c|}{$\begin{array}{c}\text { Meana }^{\mathrm{a}} \\
\begin{array}{c}\text { 95\% Confidence } \\
\text { Interval }\end{array}\end{array}$}} \\
\hline & & & & \\
\hline & & & $\begin{array}{l}\text { Lower } \\
\text { Bound }\end{array}$ & $\begin{array}{l}\text { Upper } \\
\text { Bound }\end{array}$ \\
\hline Omentum & 76.773 & 3.875 & 69.179 & 84.368 \\
\hline Flap & 84.940 & 2.833 & 79.388 & 90.492 \\
\hline Overall & 80.427 & 2.527 & 75.475 & 85.380 \\
\hline
\end{tabular}

\begin{tabular}{|c|c|c|c|c|}
\hline \multirow{2}{*}{ Surgery } & Total & \multirow{N}{*}{$\begin{array}{c}\text { N of } \\
\text { Nents }\end{array}$} & \multicolumn{2}{|c|}{ Censored } \\
\cline { 3 - 5 } & $\mathbf{N}$ & $\mathbf{N}$ & Percent \\
\hline Omentum & 62 & 12 & 50 & $80.6 \%$ \\
\hline Flap & 50 & 3 & 47 & $94.0 \%$ \\
\hline Overall & 112 & 15 & 97 & $86.6 \%$ \\
\hline \multicolumn{4}{|c}{ Case Processing Summary } \\
\hline
\end{tabular}

Age, Comorbid conditions and sepsis/hypotension at presentation affected survival rates equally among both groups.

Overall survival was $80 \%$ with omental patch closure and $94 \%$ with GSM flap closure.

The above results were statistically significant.

\section{DISCUSSION}

Cases have been encountered with late presentations and extensive peritoneal contamination leading on to omental caking/unhealthy omentum. Such cases have traditionally been treated surgically by Primary Closure/Dead Omental Patch/Jejunal Serosal Patch depending on the size of perforation. Thal's serosal patch has been used for large perforation. However, for small/medium-sized perforations, gastric seromuscular flap can be advantageous to that of a dead omental patch.

Rarely some cases may be encountered with absent omentum due to previous surgery (e.g., omentectomy in case of colectomy, obstructed/strangulated hernias). In such cases, this method will be of much benefit.

Studies were performed to see if gastric seromuscular patch elevation is anatomically feasible and to estimate the maximum size of the patch relative to its blood supply.1,2,3 A flap composed of greater omentum and a full-thickness segment of the greater curvature of the stomach, based on the right gastroepiploic artery, was first described in 1977 by
Yuzuru Kamei and Shuhei Torii. The flaps were used to treat patients with chronic soft tissue defects, ${ }^{1}$ Miller JI, Mansour, $\mathrm{KA}$, Nahai, $\mathrm{F}$ et al described management of intrathoracic defects by gastric seromuscular flaps. ${ }^{4}$ Seromuscular gastrocystoplasty lined with urothelium have been used for bladder augmentation. Surgeons have used the gastric wall from which the mucosa and submucosa had been removed.

Studies have been conducted on rats to evaluate the efficacy of repair of a large duodenal defect by a pedicled gastric seromuscular flap. ${ }^{3}$

New mucosal barrier overlaying the patch appeared to be satisfactory. All rats survived.

Cases of postpneumonectomy bronchopleural fistula treated using a gastric seromuscular and omental pedicle flap have been reported which maintained good postoperative respiratory function.1,5,6 Gastro-omental free flaps have been used in head and neck reconstruction, to close ${ }^{7}$ defects in the trachea, pharynx, cervical oesophagus and unfavourable hypopharynx. ${ }^{8}$

So this study was conducted using gastric seromuscular flap to close duodenal defects taking into account that the patch would provide an anatomical barrier to the defect developing a satisfactory new mucosal barrier compared to that of omental patching.

\section{CONCLUSION}

Both omental patch closure and GSM flap have similar survival outcomes in case of healthy omentum. In case of unhealthy or absent omentum, GSM flap has a better survival rate.

\section{REFERENCES}

1. Kamei Y, Torii S. A new composite gastric seromuscular and omental pedicle flap. Annals of Surgery 1994;220(1):97-101.

2. Aslan A, Akkaya B, Karagüzel G, et al. Bladder augmentation with an omental pedicled gastric seromuscular flap without the necessity of gastric resection. Urol Res 2004;32(4):298-303.

3. Aslan A, Elpek O. The repair of a large duodenal defect by a pedicled gastric seromuscular flap. Surg Today 2009;39(8):689-94.

4. Miller JI, Mansour KA, Nahai F, et al. Single-stage complete muscle flap closure of the postpneumonectomy empyema space: a new method and possible solution to a disturbing complication. Ann Thorac Surg 1984;38(3):227-31.

5. Patel RS, Gilbert RW. Utility of the gastro-omental free flap in head and neck reconstruction. Curr Opin Otolaryngol Head Neck Surg 2009;17(4):258-62.

6. Kondo R, Seki T, Hanamura N, et al. Gastric seromuscular and omental pedicle flap for bronchopleural fistula after pneumonectomy. The Japanese Journal of Thoracic and Cardiovascular Surgery 2000;48(8):536-9.

7. Papachristou D, Fortner JG. Experimental use of a gastric flap on an omental pedicle to close defects in the trachea, pharynx, or cervical esophagus. Plast Reconstr Surg 1977;59(3):382-5.

8. Chahine KA, Chaffanjon P, Bettega G, et al. Gastro-omental free flap in the reconstruction of the unfavourable hypopharyngeal defects: a functional assessment. J Plast Reconstr Aesthet Surg 2009;62(11):1367-73. 